\title{
A Comprehensive Review of the Diagnostic and Treatment Methods for Ovarian Cancer
}

\author{
Michelle Davis
}

\begin{abstract}
Ovarian cancer is amongst the most lifethreatening malignancy of the female reproductive system, whereas $90 \%$ of those ovarian cancers are epithelial with an overall poor five-year survival rate of $44 \%$ across all stages and all races [1]-[2], [31]. This paper aims to review the current treatment and diagnostic strategies for ovarian cancer [3]. Using grounded substantial research, multiple figures were developed to show the relations of ovarian cancer diagnostics and ovarian cancer therapeutics. It is a great start to look into what may be causing most patients to become resistant to the current standard of care, platinum-based chemotherapeutics, for ovarian cancer [4]. A comprehensive literature review will be used to understand the genetic basis of the disease and possible cancer growth patterns, so we could possibly introduce better diagnostics and therapeutics [5]. The findings show that there are a variety of treatments options other than the standard of care, platinum-based therapy [6]. Nanoparticle encapsulation therapy is one way that has been approved by the FDA to therapeutically treat ovarian cancer without the platinum resistant side effects [7]. Also, the discovery of different diagnostics for ovarian cancer can help with better individualized treatments for patients with different forms of ovarian cancer [8]. Currently, the only serous diagnostic test for the detection of ovarian cancer is high levels of Cancer Antigen 125 (CA-125), which is only shown in $50 \%$ of early staged ovarian cancers [16]. The main treatment option for ovarian cancer is platinum-based drugs, in which most cases of patients with ovarian cancer will become resistant. Detecting and treating ovarian cancer while the cells are small, contained, and still in the early stages in vivo still remains to be a challenge [9]. Here, we will demonstrate the bioelectrical interactions of the ovarian cancer cells fused with the magnetic iron oxide nanoparticles with the use of an MRI. The findings demonstrate that the diagnostic method for the early detection of epithelial ovarian cancer requires the use of magnetic iron oxide nanoparticles with specific ligand external profiles as a contrast reagent to make the small-sized ovarian cancer cells appear more visible under MRI.
\end{abstract}

Index Terms-Ovarian cancer; Platinum-based Therapy; Biomarker diagnostics; Imaging; Nanotherapeutics

\section{INTRODUCTION}

There is a growing number of literature on the early detection of epithelial ovarian cancer cells [9]. Due to the epithelium of ovaries being deep within the body, early detection holds high significance especially when preventing ovarian cancer cell from spreading. In one third of patients with epithelial ovarian cancer, the primary tumor is found to release tumor cells that circulate and metastasize throughout the circulatory system. The primary tumors quintessential phenotypes and genotypes influences specific characteristics that help us to identify why the tumor may display such exponential growth and rapid metastasis in new microenvironments [10].

The proposition of screening strategies in the early detection of patients with epithelial ovarian cancer includes biomarkers that have been shown to possibly be a better alternative to current testing using the transvaginal ultrasound and serum testing for the Cancer Antigen 125 alone, which is currently approved by the US FDA to report the manifestations of epithelial ovarian cancer progression [11]. Both of these screening strategies have been deemed and shown by a multitude of studies to be slightly unreliable due the false-positive nature of CA 125 and the low specificity of the ultrasound [7], [11]. It has also been demonstrated that biomarker profiling can help with individualized treatments for patients with epithelial ovarian cancer by predicting better treatment outcomes and patient responses [12]. An overall improvement in survival rates will be seen when there is the development of a biomarker test that has high specificity, high sensitivity, and that can detect a variety of ovarian cancers of the epithelial origin within the early stages [13], [25].

When diagnosing a high-grade ovarian malignancy, further evaluation through different forms of diagnostic measures such as the use of magnetic resonance imaging (MRI), should be taken, especially when dealing with patients of a variety of ages. Magnetic iron oxide-based nanoparticles (MNPs) are a great area of interest in the field of ovarian cancer due to its compatibility with human blood and tissue, magnetic resonance imaging reagent contrast abilities, and its responsivity to a variation of magnetic fields [15]. In order for ovarian cancer cells to appear more visible under diagnostic imaging tools, nanoparticles enhanced with targeting ligands can be used so as to enhance cellular internalization. There is a growing number of research in regard to the use of MNPs formulations coupled with MRI, however, very little research has been done when MNPs are coupled with ovarian cancer cells.

\section{RESEARCh METHOD}

\section{Review centric research}

Using recently combined research methods e.g. [66] Ovarian cancer line SK-OV-3 obtained from ATCC with known genetic variability in on or more of the following: APC, CDKN2A, FAM123B, KRAS, MLH1, NRAS, PIK3CA, STK11, and TP53 would be the best option to 
culture and exposed to an iron oxide nanoparticle formulation in order to achieve a strong magnetic profile. This ovarian cancer line would be chosen due to its common genetic variability in mutations seen in most patients diagnosed with epithelial ovarian cancer.

A comprehensive literature review on the formulation (F127250) of magnetic nanoparticles coated with pluronic polymer (F-127), $\beta$-cyclodextrin (CD), and an iron oxide core, developed by previous methods, have been shown to be a good option for haemocompatibility and MRI visible targeting while delivering a therapeutic effect to cisplatin resistant ovarian carcinoma cells all in one system [17]. This study inherently used different formulations to display enhanced MRI characteristics, however, more in-depth information could possibly be attained by keeping the current formulation the same while changing the magnetic nanoparticles core size through iron loading because nanoparticle size is crucial when it comes to clinical applications, MRI contrast visibility of small-sized cells, and bioelectrical magnetization.

The research methods used in this study follows the principles outlined by [66]. More specifically, to calculate the bioelectrical interactions of the ovarian cancer cells fused with the magnetic iron oxide nanoparticles with iron cores ranging from 5.3-7 $\mathrm{nm}$ in size, the use of a Bruker Biospec imager would be ideal to use to calculate the transverse relaxation times, T2. As provided by previously done methods, transmission electron microscopy would be used to measure the size of the magnetic cores and the magnetic properties of the F127250 formulation [66]. Toxicity evaluation measured using UV-vis spectrophotometer would give specific haemocompatibility at different concentrations.

\section{EPITHELIAL OVARIAN CANCER}

It is important to know how lethal epithelial ovarian cancer is in order to find better detection and therapeutic strategies [3]. Due to substantial research that has been done in regards to the proliferation of ovarian cancer cells, it is a great start to look into what may be causing most patients to become resistant to the current standard of care, platinum-based chemotherapeutics [4]. If we could better understand the molecular basis of the disease and each patient's individualized cancer growth patterns, we could possible introduce better technology, nanotherapeutics, immunotherapies, and targeted therapies [5]. Studying the antiproliferative effects, $\mathrm{S}$ phase cycle arrest, and apoptosis in ovarian cancer cells can lead to a variety of treatments options other than the standard of care [6], [23]. Nanoparticle encapsulation therapy is one way that has been approved by the FDA to therapeutically treat ovarian cancer without the platinum resistance [7]. The discovery of different ovarian cancer cell lines can help with better individualized treatments for patients with different forms of ovarian cancer [8].

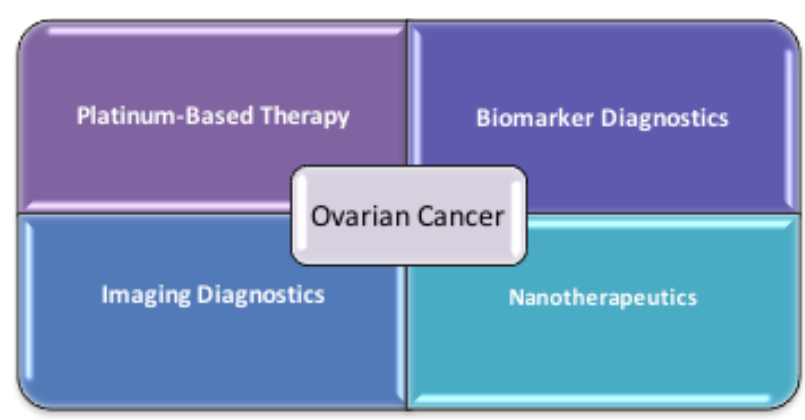

Fig. 1. Two diagnostic and two treatment methods for ovarian cancer.

\section{Platinum-BASED Therapy}

Studying the effects of platinum-based therapy on DNA can possibly help the mystery on how platinum-based drugs influences resistance [33]. The low survival rate are found by a number of factors including high resistance and relapse rates [20], [34]. Epithelial ovarian cancer shows high sensitivity to platinum based therapies compared to other cytotoxic agents [35]. It would be best to accurately record and track patient's information so it could be used in clinical trials and as a foundation to build up more knowledge to improve on noval maintenance and prognosis of ovarian cancer; especially with patients who achieved a secondary response [36]. Using natural compounds in addition to platinum-based therapies show promise when trying to overcome drug resistance due to the fact that platinum based chemotherapy causes significant damage to normal cells, tissues, and blood [37]-[38].

\section{A. Glycomarker}

The first line of treatment for ovarian cancer is platinumbased therapy [39]. Due to most patients having some sort of sensitivity and resistance to the platinum-based therapies, scientists have been looking for other markers that are easily accessible by intravenous methods that uses cost efficient materials to identify platinum-resistant cells in an effort to improve treatments [39]-[40]. Due to the differences in molecular characteristics, it is imperative to find a molecular test that can prevent the use of ineffective drugs [39].

\section{B. Platinum Sensitivity}

Studies have shown that with increasing rates of platinumbased therapies, cells become highly sensitive and begin not to react to treatment plans [41]. It has been shown that patients who have a chemotherapy relapse after six months or more are called platinum sensitive [40]. One study focused on the variation in platinum-free time intervals in which this study found that with longer platinum free intervals, patients have higher retreatment rates [41]. This study and multiple others found that there is some stratification that needs to be done when differentiating platinum resistivity and sensitivity in patients with reoccurring ovarian cancer [40]-[41].

\section{Platinum Resistance}

Unlike platinum sensitivity, platinum resistivity is described as patients who relapse within six months or less [40]. These patients also display lower response rates to continued administered chemotherapy and include a variety of disease recurrence symptoms due to common mechanisms such as annexin A3 and a number of genes, including XIAP 
and LRP [21], [27], [40]. The characteristics of platinum resistant and platinum sensitivity have a fine line in between in which patients experience a rise in CA-125 and variation in radiologic images such as PET and CT [40].

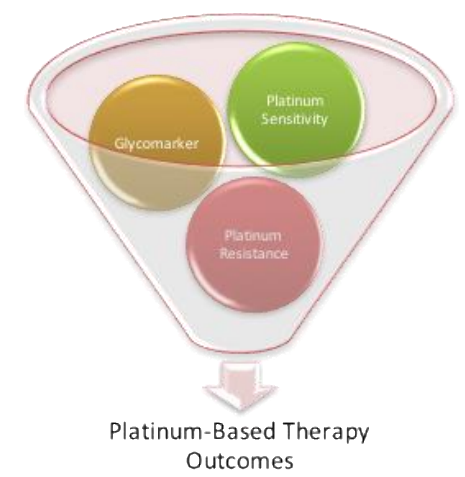

Fig. 2. Common outcomes from platinum-based therapeutics.

\section{BIOMARKER DIAGNOSTICS}

It is important to note that the use of biomarkers is the future of cancer research, more specifically, ovarian cancer research because it is able to show a better outcome that the currently used methods of platinum-based therapy [7]. Biomarker profiling can also predict better treatment outcomes and predict patient response [12]. Finding certain biomarkers for specific genes, such as the novel serum tumor antigen-associated autoantibody (TAAb), heat shock protein 10 (HSP10), and PD-1 can play a role in better detection multiple cancers, including ovarian cancer [11], [28], [32]. We also need more efficient ways of harvesting these proteins [42]. One other important thing to realize is we need to see a more specific genetic pattern in these biomarkers in order to use them for detection [43]. The elevation in this tumor would show promise towards better detection, prognosis, and faster diagnoses for patients with ovarian cancer [33].

\section{A. $C A-125$}

The CA-125 test is FDA approved and has been used for the screening and diagnosis of a variety of stages for ovarian cancer. Even though the CA-125 test is clinically approved, this test proves to have variants in specificity and sensitivity [44]. Cancer antigen 125 is a glycoprotein that is secreted into the bloodstream and has been found by a variety of studies to not only be specific to ovarian cancer, but can show elevated levels in pelvic masses such as cysts and in pregnancy, while $20 \%$ of ovarian cancers can still express no increase in those levels [11], [29], [44]. The CA-125 test is also not sensitive enough to test for early stage epithelial ovarian cancer, which is a major disadvantage due to the fact that the five-year survival rate increases, the earlier the cancer is diagnosed and treated [44].

\section{B. Tumor antigen-associated autoantibody}

One of the biomarkers, such as the novel serum tumor antigen-associated autoantibody (TAAb), which are serum biomarkers which are easily accessible from blood samples, shows promise when it comes to the early detection and diagnosis of multiple cancers, including high grade serous epithelial ovarian cancer [45]. In one study, the primary goal was to differentiate between healthy patients and patients with benign serous ovarian tumors so as to improve the sensitivity rates independent of the transvaginal ultrasound and reduce the false positive rates when combined with the CA-125 serum test [11]. Possible success with this specific biomarker may eliminate the problem of diagnosing epithelial ovarian cancer too late which is a reoccurring problem with the use of the CA-125 serum test alone because of its patientto-patient variability and the transvaginal ultrasound which cannot detect miniscule tumors in the early stages [11].

\section{Genetic Biomarkers}

One important thing to realize is that we need to see more specific genetic patterns in order to use genetic biomarkers for the screening, detection, and tumor stratification of EOC [43]. One study used samples of blood and tissue from EOC patients to determine genetic variations using NGS, microRNA patterns, and DNA methylation patterns using array technology to improve screening and detection, thus, possibly leading to an improvement in diagnosis and therapeutics [13]. Again, overcoming the variant high false positive rates from the current and standard serum CA-125 test is a major goal when utilizing genetic profiling in a number studies that label certain genes as potential biomarkers, such as the TP53 gene, the RAS protooncogenes, KRAS, HRAS, NRAS, and the BRACA genes [13], [46]-[47]. Designated type II carcinomas are premium quality serous carcinosarcomas which display rapid mutations in the tumor suppressor, TP53, unlike its counterpart, designated type I, in which TP53 mutations rarely as expressed. While TP53 remains low in low-grade tumors, the KRAS, BRAF, and ERBB2 mutations are high [48]. The mutations in the germline of BRACA1 and BRACA2 genes are the most apparent genetic risk factors in epithelial ovarian cancer, effecting 8-15 percent of women that have been diagnosed with epithelial ovarian cancer [49]. BRACA1 holds multiple roles such as DNA repair, transcriptional regulation, and mitosis. In addition, BRACA2 is used for homologous recombination. Some studies have attempted to show the manifestations between the carriers and non-carriers of BRACA1 and BRACA2 genes, however, it's relative prognosis of EOC still remains slightly unclear [50].

\section{D. $A L D H$}

The potential biomarker, ALDH, is paving the way to help identify ways to detect platinum-based drug resistance especially because it displays inherit characteristics such as multi-drug resistance and smaller size [5], [22]. Currently there are only a few biomarkers, however, the expression of ALDH1 and its relationship with cancer stem cells (CSCs), lower reaction oxygen species, and biomarker expression in pOC and rOC have been heavily investigated in two specific scientific models [51]-[52]. Scientists have also been able to combine investigative methods of ALDH as a prognostic biomarker [53]. The metabolism of ovarian cancer cells is a good indicator of the cancer cells microenvironments in comparison to normal ovarian cells [54]. In order to get a better population dynamic of the expression of ALDH in patients diagnosed with HGSC cancer, larger and more specific groups of the tumor microenvironment should be studied [55]. 


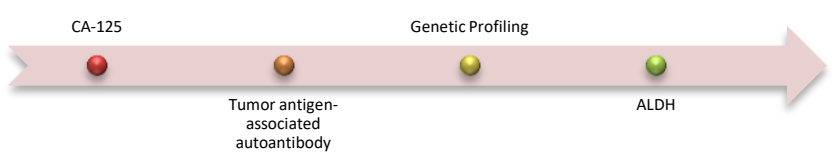

Fig. 3. Ovarian Cancer Biomarkers

\section{NANOTHERAPEUTICS}

More nanotheraputic approaches, like Doxil, should be approved by the FDA to use clinically instead of platinumbased therapies [7]. Nanotechnology can help with a combinational drug delivery system that intensifies the efficacy of the chemical drug and directly targets the ovaries without affecting the neighboring cells of the reproductive system [24], [56]. Using different tumor-targeting moieties can help the efficacy of delivering drugs to tumor cells instead of normal cells [57]. Early detection screening of ovarian cancer cells is imperative since the overall survival rate increases significantly when ovarian cancer is caught in the early stages [58]. Even though nanotechnology is still young when it comes to ovarian cancer nanotherapeutics, it shows promises when trying to target specific cancer cell lines in vivo [30], [59]. One statement to think about is how much of this combinational drug therapy would be needed to effectively target the ovarian cancer cells and not the normal or malignant cells [60].

\section{A. Doxil}

Doxil is one of the FDA-approved treatment options for laterstaged ovarian cancer that can be clinically used as a "firstline" form of therapeutics [61]. It can also be used after platinum-based chemotherapy that has failed to stop the progression, metastasis, or recurrent of ovarian cancer [61]. Doxil is the name of the prescription medicine and is a specifically coated active form of Doxorubicin, however, due to the serious side effects that are associated with doxorubicin, scientists have attempted to find other nanocarriers, such as polymeric single micelles, to encapsulate drugs as a possible alternative [61]. There are numbers of studies that show the beneficial effects of using doxorubicin in a liposomal encapsulation drug delivery vehicle [62].

\section{B. Lyophilisomes}

Due to the adverse side effects of most conventional chemotherapeutics, such as platinum based chemotherapy. lyophilisomes are drug delivery vehicles that and be classified from nano-sized to micro-sized [26], [62]. They can be prepared using proteins like album, collagen, and elastin in which a drug can be loaded inside and used to target specific cells, more specifically, ovarian cancer cells [62]. It is imperative to develop nanotherapeutic methods that target only cancerous tissues and cells while leaving healthy neighboring cells to continue to proliferate and remodel. When preparing lyophilisomes, it's walls can be functionalized with specific targeting ligands and antibodies to produce a more favorable therapeutic effects [62].

\section{IMAGING DiAGNOSTICS}

The diagnosis of epithelial ovarian cancer is usually found in $80 \%$ of later staged cases when the cancer has already metastasized and survival rates are less than $20 \%$. When the cancer is diagnosed in the earlier stages, the five-year survival rate is $85 \%$ [63]. The diagnostic evaluation follows after numerous imaging measures and is a significant process which includes a combination of tests in order to classify, stratify, and identify epithelial ovarian high-grade malignancies

\section{A. MRI}

When diagnosing a high-grade ovarian malignancy, further evaluation through different forms of diagnostic measures should be taken especially dealing with patients of a variety of ages. The use of contrast MRI in the prospective diagnosis can be used to further investigate the sensitivity and specificity, where MRI can be used as a valuable tool to detect the diagnostic criteria such as wall thickness greater than $3 \mathrm{~mm}$, cancer cell local invasion, morphology, septal thickness, and necrosis of epithelial ovarian tumors because it has a greater soft tissue contrast than a CT scan [14].

\section{B. ${ }^{18} F-F D G P E T / C T$}

The ${ }^{18}$ F-FDG PET/CT sensitivity (52-58\%), high falsenegative results, high false-positive results, and specificity (76-78\%) is not recommended for the initial detection of epithelial ovarian cancer. This form of diagnostic testing is found to be more useful as a postoperative susceptive reoccurrence follow-up especially when PET is combined with contrast-enhanced CT in order to stratify malignancy stages and improved accuracy, sensitivity (up to 91\%), and specificity (up to 100\%) [14], [64]. An example of this can be seen in one small cohort study of 41 participants with ovarian cancer reoccurrence; it was found that ${ }^{18} \mathrm{~F}-\mathrm{FDG} \mathrm{PET} / \mathrm{CT}$ is more accurate than CE/CET alone with values of $92 \%$ vs. $73 \%, 90 \%$ vs. $55 \%$, and $91 \%$ vs $63 \%$ for sensitivity, specificity, and accuracy when detecting for the recurrence of ovarian cancer [65].

\section{C. $M N P S$}

A formulation (F127250) of magnetic nanoparticles coated with pluronic polymer (F-127), $\beta$-cyclodextrin (CD), and an iron oxide core, developed by previous methods, have been shown to be a good option for haemocompatibility and MRI visible targeting while delivering a therapeutic effect to cisplatin resistant ovarian carcinoma cells all in one system [17]. Cai et al was able to demonstrate that an increase in magnetization, contrast ability, and relaxation times were shown with an increase in size of ferrimagnetic cores, with, $5.3 \mathrm{~nm}$ in size being the strongest [66]. Another important aspect is the interactions of these MNPs in human blood circulation due to it being the main nanoparticles locative route. Previous methods have taken safety measures when nanoparticle formulations have been mixed with human blood [17]. 


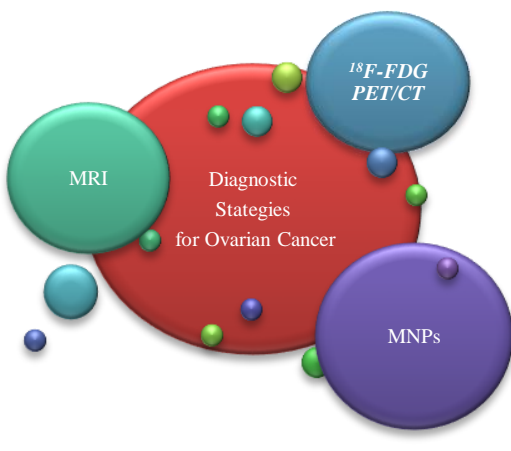

Fig. 4. Ovarian Cancer Diagnostic Strategies

\section{DISCUSS RESEARCH}

Due to the fact that epithelial ovarian cancer is one of the most common cancers of the female gynecological system, it is imperative to develop and identify a variety of diagnostic tools to provide accurate and detailed histological information by the way of serological testing or imaging. However, the transvaginal ultrasound, the current diagnostic biomarker testing assays, MMP-7, CCL18, CCL11, and the FDA-approved human Epididymis protein 4 marker, OVA1 test, ROMA, and the CA-125 assays, are typically not used by themselves because each test does not provide sufficient information about sensitivity, specificity, early detection, and specific tumor characteristics such as differentiating between benign, borderline, or malignant tumors. When combining other imaging sources and variables with the current clinical standard, such as computed tomography and magnetic resonance imaging, these can be introduced to provide locative and morphological distinctive features common to ovarian tumors to definitively be used for diagnosis. Since epithelial ovarian cancer is typically found in the later stages after the cancer has metastasized, new and improved diagnostic standards must be applied to dramatically increase the 5-year survival rate, prognosis, and clinical trial outcomes.

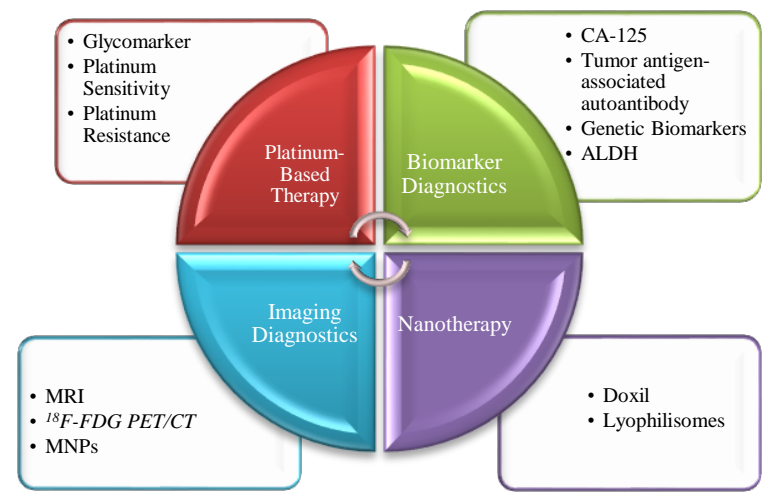

Fig. 5. Review of diagnostic and therapeutic strategies for ovarian cancer.

\section{RESULTS AND DISCUSSION}

There is a growing number of literary sources that have utilized magnetic iron oxide-based nanoparticles (MNPs) with carcinoma cells because of its biocompatibility, magnetic resonance imaging (MRI) reagent contrast abilities, responsiveness to alternating magnetic fields (AMF), and adherence to regular magnetic fields [16], [32]. When designing a nanoparticle for the diagnosis of early high grade ovarian cancer, there should be major considerations taken when including surface modifications with specific ligand external profiles so as to enhance cellular internalization and make the ovarian cancer cells appear more visible under MRI [7]. There are a variety of studies covering the use of MNPs formulations in conjunction with MRI, however, there is a lack of research pertaining to the bioelectrical interactions of ovarian cancer cells with the MNPs formulation and MRI [17].

Cai et al was able to demonstrate that an increase in magnetization, contrast ability, and relaxation times were shown with an increase in size of ferrimagnetic cores, with, $5.3 \mathrm{~nm}$ in size being the strongest [65]. Increasing the iron cores up to $7 \mathrm{~nm}$ in size would show to be an even higher increase in saturation magnetization and possible detect SK$\mathrm{OV}-3$ ovarian cancer cells lower than $10^{4}$ cells $\mathrm{mL}^{-1}$. Another important aspect is the interactions of these MNPs in human blood circulation due to it being the main nanoparticles locative route. Previous methods have demonstrated nanoparticle formulations being non-toxic to human blood, however, other formulations at $30 \mu \mathrm{g}$ would show high toxicity, clumping, and a rapid change in red blood cell morphological studies [17]. One study used $100 \mu$ of human blood and incubated the formulations for $2 \mathrm{hrs}$. A possible change could be seen when incubation is increased to 24 and 48 hours for a more realistic reading.

\section{CONCLUSION}

It is imperative to develop a novel diagnostic method to determine the early stages of epithelial ovarian cancer that can possibly replace the main diagnostic standards, the CA125 test and the transvaginal ultrasound, due to their unreliability, variability, low specificity, low sensitivity, and false-positive nature. The use of contrast MRI agents have been shown in the literature to have the most promise when representing specific diagnostic criteria for regular carcinoma cells. Magnetic nanoparticles have been shown to have biocompatibility, therapeutic characteristics, MRI magnetic contrast reagents, and haemocompatibility, however, the bioelectrical interactions of SK-OV-3 human ovarian cancer cells with the F127250 formulation have not been studied in the past. These interactions are very crucial, especial when it comes to diagnostic and therapeutic approaches in clinical aspects and should be explored in a more through matter. Minor changes such as a slight increase in iron cores leading to higher carcinoma cell detectability, enhanced magnetization shown through transverse relaxation times, an increase in incubation times for the haemocompatibility, and toxicity analysis would be unique and readily transferrable to clinical applications [19].

\section{ACKNOWLEDGMENT}

Thanks to Professor Christian Bach and Viraj Banpel.

\section{REFERENCES}

[1] Kim, A., et al., Therapeutic strategies in epithelial ovarian cancer. Journal of experimental \& clinical cancer research, 2012. 31(1): p. 14. 
[2] Toss, A., et al., Hereditary ovarian cancer: not only BRCA 1 and 2 genes. BioMed research international, 2015. 2015.

[3] Oronsky, B., et al., A brief review of the management of platinumresistant-platinum-refractory ovarian cancer. Medical Oncology, 2017. 34(6): p. 103.

[4] Grunewald, T. and J.A. Ledermann, Targeted therapies for ovarian cancer. Best Practice \& Research Clinical Obstetrics \& Gynaecology, 2017. 41: p. 139-152.

[5] Januchowski, R., et al., Inhibition of ALDH1A1 activity decrease expression of drug transporters and reduces chemotherapy resistance in ovarian cancer cell lines. The international journal of biochemistry \& cell biology, 2016. 78: p. 248-259.

[6] Wang, Y., et al., Anti-proliferative effect and cell cycle arrest induced by saponins extracted from tea (Camellia sinensis) flower in human ovarian cancer cells. Journal of Functional Foods, 2017. 37: p. 310321.

[7] Engelberth, S.A., N. Hempel, and M. Bergkvist, Development of nanoscale approaches for ovarian cancer therapeutics and diagnostics. Critical ReviewsTM in Oncogenesis, 2014. 19(3-4).

[8] Kreuzinger, C., et al., Molecular characterization of 7 new established cell lines from high grade serous ovarian cancer. Cancer letters, 2015. 362(2): p. 218-228

[9] Davies, S., et al., High incidence of ErbB3, ErbB4 and MET expression In ovarian cancer. International journal of gynecological pathology: official journal of the International Society of Gynecological Pathologists, 2014. 33(4): p. 402

[10] Van Berckelaer, C., et al., Current and future role of circulating tumor cells in patients with epithelial ovarian cancer. European Journal of Surgical Oncology (EJSO), 2016. 42(12): p. 1772-1779.

[11] Katchman, B.A., et al., Autoantibody biomarkers for the detection of serous ovarian cancer. Gynecologic Oncology, 2017. 146(1): p. 129136.

[12] Herzog, T.J., et al., Impact of molecular profiling on overall survival of patients with advanced ovarian cancer. Oncotarget, 2016. 7(15): p. 19840

[13] Hentze, J.L., et al., Searching for new biomarkers in ovarian cancer patients: Rationale and design of a retrospective study under the Mermaid III project. Contemporary Clinical Trials Communications, 2017. 8: p. 167-174.

[14] Iyer, V.R. and S.I. Lee, MRI, CT, and PET/CT for Ovarian Cancer Detection and Adnexal Lesion Characterization. American Journal of Roentgenology, 2010. 194(2): p. 311-321.

[15] Wabler, M., et al., Magnetic resonance imaging contrast of iron oxide nanoparticles developed for hyperthermia is dominated by iron content. International journal of hyperthermia : the official journal of European Society for Hyperthermic Oncology, North American Hyperthermia Group, 2014. 30(3): p. 192-200.

[16] Das, P. M., \& Bast, R. C. (2008). Early detection of ovarian cancer. Biomarkers in Medicine, 2(3), 291-303. http://doi.org/10.2217/17520363.2.3.291

[17] Yallapu, M.M., et al., Multi-functional Magnetic Nanoparticles for Magnetic Resonance Imaging and Cancer Therapy. Biomaterials, 2011. 32(7): p. 1890-1905

[18] LePine, J.A. and A. Wilcox-King, Developing novel theoretical insight from reviews of existing theory and research. Academy of Management Review, 2010. 35(4): p. 506-509.

[19] Mikulka, J., \& Dvorák, P. (2014). Fast Calculation of T2 Relaxation Time in Magnetic Resonance Imaging. Session 4A12 SC1: Extended/Unconventional Electromagnetic Theory, Electrohydrodynamics/Electro-magneto-hydrodynamics, and Electro-biology, 1860.

[20] Vaughan, S., Coward, J. I., Bast Jr., R. C., Berchuck, A., Berek, J. S., Brenton, J. D., ... Balkwill, F. R. (2011). Rethinking Ovarian Cancer: Recommendations for Improving Outcomes. Nature Reviews. Cancer, 11(10), 719-725. http://doi.org/10.1038/nrc3144

[21] Yin, J., Yan, X., Yao, X., Zhang, Y., Shan, Y., Mao, N., ... Pan, L. (2012). Secretion of annexin A3 from ovarian cancer cells and its association with platinum resistance in ovarian cancer patients. Journal of Cellular and Molecular Medicine, 16(2), 337-348.

[22] Sharrow, A.C., et al., Characterization of aldehyde dehydrogenase 1 high ovarian cancer cells: Towards targeted stem cell therapy. Gynecologic Oncology, 2016. 142(2): p. 341-348.

[23] Chen, S., et al., Fascaplysin inhibit ovarian cancer cell proliferation and metastasis through inhibiting CDK4. Gene, 2017. 635(Supplemen C): p. 3-8.

[24] Geninatti Crich, S., et al., "Theranostic" nanoparticles loaded with imaging probes and rubrocurcumin for a combined cancer therapy by folate receptor targeting. 2017.

[25] Tajmul, M., Parween, F., Singh, L., Mathur, S. R., Sharma, J. B. Kumar, S., ... \& Yadav, S. (2018). Identification and validation of salivary proteomic signatures for non-invasive detection of ovarian cancer. International journal of biological macromolecules, 108, 503 514.

[26] van Bracht, E., Raavé, R., Verdurmen, W. P., Wismans, R. G., Geutjes, P. J., Brock, R. E., ... \& Daamen, W. F. (2012). Lyophilisomes as a new generation of drug delivery capsules. International journal of pharmaceutics, 439(1), 127-135.

[27] Wang, Y., et al., HPIP expression predicts chemoresistance and poor clinical outcomes in patients with epithelial ovarian cancer. Human Pathology, 2017. 60(Supplement C): p. 114-120.

[28] Chapman, J.S., et al., Immunoprofiling epithelial ovarian cancer. Gynecologic Oncology, 2017. 147(1): p. 219.

[29] Fruscio, R., et al., Ovarian cancer in pregnancy. Best Practice \& Research Clinical Obstetrics \& Gynaecology, 2017.41(Supplement C) p. 108-117.

[30] Lee, M., et al., Predictive value of circulating tumor cells (CTCs) captured by microfluidic device in patients with epithelial ovarian cancer. Gynecologic Oncology, 2017. 145(2): p. 361-365.

[31] Pistollato, F., et al., The use of natural compounds for the targeting and chemoprevention of ovarian cancer. Cancer Letters, 2017.

[32] Boya, V.N., et al., Probing mucin interaction behavior of magnetic nanoparticles. Journal of Colloid and Interface Science, 2017 488(Supplement C): p. 258-268.

[33] Chen, J.B., M.A. Neves, and M. Thompson, Biosensor surface attachment of the ovarian cancer biomarker HSP10 via His-tag modification. Sensing and Bio-Sensing Research, 2016. 11: p. 107-112.

[34] Luvero, D., A. Milani, and J.A. Ledermann, Treatment options in recurrent ovarian cancer: latest evidence and clinical potential. Therapeutic advances in medical oncology, 2014. 6(5): p. 229-239.

[35] Bookman, M.A., et al., Evaluation of new platinum-based treatment regimens in advanced-stage ovarian cancer: a Phase III Trial of the Gynecologic Cancer Intergroup. Journal of Clinical Oncology, 2009. 27(9): p. 1419-1425

[36] Markman, M., et al., Duration of response to second-line, platinumbased chemotherapy for ovarian cancer: implications for patient management and clinical trial design. Journal of Clinical Oncology, 2004. 22(15): p. 3120-3125.

[37] Anwar, M.S., et al., Natural compounds alone and in combination with platinum drugs found to show significant anti-tumour activity against ovarian cancer cell lines. 2017, AACR

[38] Flanagan, J.M., et al., Platinum-Based Chemotherapy Induces Methylation Changes in Blood DNA Associated with Overall Survival in Patients with Ovarian Cancer. Clinical Cancer Research, 2017. 23(9): p. 2213-2222.

[39] Razi, N., et al., Abstract TMEM-035: GLYCOMARKERS FOR PREDICTING PLATINUM-DRUG RESPONSE IN OVARIAN CANCER. 2017, AACR

[40] Davis, A., A.V. Tinker, and M. Friedlander, "Platinum resistant" ovarian cancer: What is it, who to treat and how to measure benefit? Gynecologic Oncology, 2014. 133(3): p. 624-631.

[41] Alvarez, R.D., et al., Moving beyond the platinum sensitive/resistan paradigm for patients with recurrent ovarian cancer. Gynecologic Oncology, 2016. 141(3): p. 405-409.

[42] Chatterjee, M., L.C. Hurley, and M.A. Tainsky, Paraneoplastic antigens as biomarkers for early diagnosis of ovarian cancer. Gynecologic Oncology Reports, 2017.

[43] Gloss, B.S. and G. Samimi, Epigenetic biomarkers in epithelial ovarian cancer. Cancer letters, 2014. 342(2): p. 257-263.

[44] Park, Y., et al., Diagnostic performances of HE4 and CA125 for the detection of ovarian cancer from patients with various gynecologic and non-gynecologic diseases. Clinical Biochemistry, 2011. 44(10): p. 884 888.

[45] Heo, C.-K., Y.Y. Bahk, and E.-W. Cho, Tumor-associated autoantibodies as diagnostic and prognostic biomarkers. BMB Reports, 2012. 45(12): p. 677-685

[46] Rechsteiner, M., et al., TP53 mutations are common in all subtypes of epithelial ovarian cancer and occur concomitantly with KRAS mutations in the mucinous type. Experimental and Molecular Pathology, 2013. 95(2): p. 235-241.

[47] Vanderstichele, A., et al., Genomic signatures as predictive biomarkers of homologous recombination deficiency in ovarian cancer. European Journal of Cancer, 2017. 86(Supplement C): p. 5-14.

[48] Kurman, R.J. and I.-M. Shih, The Origin and Pathogenesis of Epithelial Ovarian Cancer- a Proposed Unifying Theory. The American journal of surgical pathology, 2010. 34(3): p. 433-443.

[49] Maistro, S., et al., Germline mutations in BRCA1 and BRCA2 in epithelial ovarian cancer patients in Brazil. BMC Cancer, 2016. 16: $\mathrm{p}$ 934. 
[50] Bolton, K.L., et al., Association between BRCA1 and BRCA2 mutations and survival in women with invasive epithelial ovarian cancer. Jama, 2012. 307(4): p. 382-389.

[51] Mizuno, T., et al., Cancer stem-like cells of ovarian clear cell carcinoma are enriched in the ALDH-high population associated with an accelerated scavenging system in reactive oxygen species. Gynecologic Oncology, 2015. 137(2): p. 299-305.

[52] Ruscito, I., et al., Exploring the clonal evolution of CD133/aldehydedehydrogenase-1 (ALDH1)-positive cancer stem-like cells from primary to recurrent high-grade serous ovarian cancer (HGSOC). A study of the Ovarian Cancer Therapy-Innovative Models Prolong Survival (OCTIPS) Consortium. European Journal of Cancer, 2017. 79 p. 214-225.

[53] Roy, M., et al., Aldehyde dehydrogenase 1 (ALDH1A1) expression by immunohistochemistry is associated with chemo-refractoriness in patients with high-grade ovarian serous carcinoma. Human pathology, 2017.

[54] Orywal, K., et al., The activity of class I, II, III and IV alcohol dehydrogenase isoenzymes and aldehyde dehydrogenase in ovarian cancer and ovarian cysts. Advances in medical sciences, 2013. 58(2): p. 216-220.

[55] Liebscher, C.A., et al., Aldehyde dehydrogenase 1/epidermal growth factor receptor coexpression is characteristic of a highly aggressive, poor-prognosis subgroup of high-grade serous ovarian carcinoma. Human pathology, 2013. 44(8): p. 1465-1471.

[56] van der Steen, S.C., et al., Targeting the extracellular matrix of ovarian cancer using functionalized, drug loaded lyophilisomes. European Journal of Pharmaceutics and Biopharmaceutics, 2017. 113: p. 229239.

[57] Sapiezynski, J., et al., Precision targeted therapy of ovarian cancer. Journal of Controlled Release, 2016. 243: p. 250-268.

[58] Kim, P.S., S. Djazayeri, and R. Zeineldin, Novel nanotechnology approaches to diagnosis and therapy of ovarian cancer. Gynecologic Oncology, 2011. 120(3): p. 393-403.

[59] $\mathrm{Pi}, \mathrm{F}$., et al., RNA nanoparticles harboring annexin A2 aptamer can target ovarian cancer for tumor-specific doxorubicin delivery. Nanomedicine: Nanotechnology, Biology and Medicine, 2017. 13(3): p. 1183-1193.

[60] Koutsaki, M., D.A. Spandidos, and A. Zaravinos, Epithelialmesenchymal transition-associated miRNAs in ovarian carcinoma, with highlight on the miR-200 family: Prognostic value and prospective role in ovarian cancer therapeutics. Cancer letters, 2014. 351(2): p 173-181.

[61] Cagel, M., et al., Mixed micelles for encapsulation of doxorubicin with enhanced in vitro cytotoxicity on breast and ovarian cancer cell lines

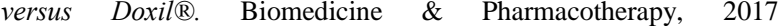
95(Supplement C): p. 894-903.

[62] van der Steen, S.C.H.A., et al., Targeting the extracellular matrix of ovarian cancer using functionalized, drug loaded lyophilisomes. European Journal of Pharmaceutics and Biopharmaceutics, 2017. 113(Supplement C): p. 229-239.

[63] Skates, S.J., OCS: Development of the Risk of Ovarian Cancer Algorithm (ROCA) and ROCA screening trials. International journal of gynecological cancer: official journal of the International Gynecological Cancer Society, 2012. 22(Suppl 1): p. S24-S26.

[64] Foti, P.V., et al., MR imaging of ovarian masses: classification and differential diagnosis. Insights into Imaging, 2016. 7(1): p. 21-41.

[65] Abdelhafez, Y., et al., Role of $18 F-F D G$ PET/CT in the detection of ovarian cancer recurrence in the setting of normal tumor markers. The Egyptian Journal of Radiology and Nuclear Medicine, 2016. 47(4): p. 1787-1794.

[66] Cai, Y., et al., Enhanced magnetic resonance imaging and staining of cancer cells using ferrimagnetic H-ferritin nanoparticles with increasing core size. International Journal of Nanomedicine, 2015. 10: p. 2619-2634.

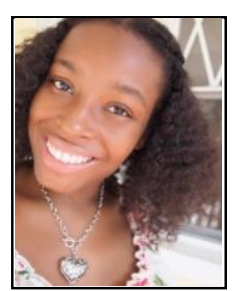

Michelle Davis was born in Westchester, New York and is currently a first-year biomedical engineering Masters student at the University of Bridgeport in Bridgeport, Connecticut. She obtained her Bachelor of Science Degree in Human biology from the University at Albany where she concentrated in chemistry and human anatomy and physiology.

Michelle has obtained her previous clinical work experience from New York University Langone Medical Center where she was invited to train under the tutelage of the professor and former chairman of the NYU Department of Psychiatry in order to gain a deeper insight into biomedical research by witnessing how to screen, enroll, and track study participants. She also worked with Reproductive Medicine Associates of New York where she practiced the role of patient services coordinator, financial guide, and medical chaperone in collaboration with a multidisciplinary team of boardcertified reproductive endocrinologists in efforts to sustain a continuum of successful fertility treatments with compassionate and individualized care. She is currently a member of the Biomedical Engineering Society and Engineers without Borders Society at the University of Bridgeport where she is gaining experience to epitomize an engineer's role to improve healthcare diagnosis, treatment, and the design of medical devices. 